\title{
Modelos científicos y algunas implicaciones en la formación inicial de profesores de química
}

\author{
Quira A. Sanabria Rojas* \\ Royman Pérez Miranda** \\ Rómulo Gallego Badillo***
}

Artículo recibido: 20-11-2008 y aprobado: 17-08-2009

\begin{abstract}
Scientific models and some implications in chemistry's teachers in initial training
\end{abstract}

Resumen: En este artículo se presentan algunas reflexiones y resultados de un estudio realizado con estudiantes de Licenciatura en Química de la Universidad Pedagógica Nacional de Bogotá. El objetivo principal del trabajo se orientó a la reconstrucción en el aula, de los modelos científicos para las disoluciones electrolíticas en medio acuoso. Uno de los propósitos de este trabajo fue revisar y analizar la pertinencia de un espacio académico en la formación profesional del profesor de Química, en el cual se tengan en cuenta las elaboraciones hechas por los estudiantes en los espacios académicos de la Licenciatura en relación con este objeto de estudio; la transformación de las representaciones que tienen los futuros profesores cuando se ponen en contraposición con documentos publicados en torno a la temática mencionada; y, las aproximaciones epistemológicas sobre la categoría de modelos científicos planteada en la literatura actual del tema. Los resultados obtenidos hablan en favor de las estrategias didácticas empleadas en este espacio académico, puesto que propiciaron la transformación de los modelos de sentido común que tenían los estudiantes al ingresar al proceso de formación, al pasar a una aproximación del modelo analógico - simbólico de las Disoluciones electrolíticas en medio acuoso.

Palabras clave: Formación inicial de profesores de química, modelos científicos, didáctica de las ciencias, disoluciones electrolíticas.
Abstract: In this writing it is presented findings about a research carried out with Chemistry's teachers in initial formation of the department of Chemistry from Universidad Pedagógica Nacional from Bogota. The main objective was the reconstruction in the classroom of scientific models for electrolytic dissolutions in aqueous medium. The purpose was to revise and to analyse the pertinence of starting a professional training from spaces focus on studies like those; also the permanence or modification of some representations taking into account previous research projects and some epistemological approaches that specialists have proposed for the category of scientific models. The final results are in favour of the implemented strategies since they help the teachers in initial training to change their common sense models and finally to approach to an analogicalsymbolic model about electrolitytic dissolution.

Key words: Initial formation of chemistry teachers, scientific models, didactic of the sciences.

\footnotetext{
* Profesor Universidad Pedagógica Nacional. Departamento de Química. Correo electrónico: qsanabria@pedagogica.edu.co

** Profesor Universidad Pedagógica Nacional. Departamento de Química. Correo electrónico: royman@pedagogica.edu.co

*** Profesor Universidad Pedagógica Nacional. Departamento de Química. Correo electrónico: rgallego@pedagogica.edu.co
} 


\section{Introducción}

En la actualidad ha cobrado importancia la estructuración y consolidación de los soportes epistemológicos y didácticos de la Química durante los procesos de formación de profesores, puesto que de ello depende la concepción de enseñanza de las ciencias y de la química que es capaz de construir el profesional en ejercicio, y que orienta las decisiones que toma. Superando el paradigma puramente metodológico, la didáctica de las ciencias ha consolidado ya varias líneas de investigación como: historia y epistemología, evaluación, relaciones CTSA, formación de profesores y representaciones o modelos científicos. Este trabajo se enmarca en esta última línea, sin dejar de reconocer otros aportes que se hacen desde las otras líneas, que la hacen compleja y la soportan.

Se considera que la elaboración de modelos es la ruta más efectiva para obtener explicaciones del entorno, los modelos son abstractos y reducidos y dan cuenta sólo de una parte de la realidad, pero al combinarse con otros, permiten reconocer la complejidad en las explicaciones que se dan sobre la naturaleza (Gieré, 1992). Los modelos científicos, tienen un componente epistemológico fuerte, el cual en palabras de Gieré, está relacionado con la interpretación e identificación de una representación presente al momento de usar el lenguaje para describir los objetos de la realidad. Sin embargo, cuando las representaciones son de carácter científico, éstas son válidas para un colectivo, dentro de los criterios de realidad que sean atribuidos por dicha comunidad de especialistas. Hacer un análisis de cómo se van modificando los modelos sobre un contexto específico de los profeso- res en formación de química, permite reconocer obstáculos epistemológicos producto del proceso escolar vivido por el profesor en formación en instancias anteriores (Pessoa de Carvalho, 2004; Gallego Badillo, 2004; Gil Pérez, Carrascosa Alís, Martínez Terrades, 1999). A su vez, muestra las barreras que posiblemente hay que sortear para que los modelos elaborados inicien su proceso de trasformación hacia niveles complejos de interpretación del contexto en que se producen.

La química históricamente no ha considerado los fenómenos naturales como objeto de estudio, más bien se ha interesado por los modelos mentales que se relacionan entre sí con desarrollos epistemológicos particulares. Presupuestos científicos que se han construido socialmente, dentro de comunidades de especialistas, que responden a unas condiciones de carácter cultural, económico y político. Tales comunidades, elaboran sistemas representacionales que se distancian de lo cotidiano, son mucho más abstractos, y pueden mostrar diversos niveles de complejidad (Caldín, 2002). Las diferentes explicaciones que se han elaborado acerca de esa estructura y comportamiento a través de las épocas, muestran el uso de modelos de características diversas conformes con las particularidades de los problemas planteados (Aduríz-Bravo, Garófalo, Greco, y Galagovsky, 2005; Gieré, 1999).

Ha cobrado mayor importancia en la actualidad una enseñanza de las ciencias centrada en la reconstrucción en el aula de los modelos científicos convertidos en planes de estudio, dentro de los currículos. De la misma manera, existe una discusión, alrededor de lo que se quiere significar con la categoría epis- 
temológica de modelo científico (Caldín, 2002; Giere, 1999; Lombardi. 1998), que se ha extendido hasta señalar la necesidad de una reconstrucción histórica para decidir la clase o clases de modelo científico que harán posible categorizar y explicar un evento objeto de estudio (Greca y Dos santos, 2005). Al respecto, Adurìz (2006), anota que en los niveles escolares, cualquiera que sean, lo que se hace es elaborar un modelo didactizado tan válido como el científico, pero que, realizado de manera ingenua, puede generar un modelo tan reducido, que puede provocar más confusión que avance en el entendimiento de un problema de trabajo en el aula.

Recientemente, se admite la conceptualización en química soportada en la producción de modelos. Algunas de las investigaciones en didáctica han establecido que los estudiantes hacen aproximaciones significativas sobre unos modelos científicos, cuando éstos se hacen objeto de trabajo en el aula desde una perspectiva histórica (Galagovsky,et al, 2009; Niaz, 2009; De Amoré, 2007; Gallego Badillo y Pérez Miranda, 2003; Aduríz- Bravo, Izquierdo y Estanny, 2002).

Es necesario resaltar, dentro de las publicaciones que hacen parte de los antecedentes de este estudio, los trabajos de Raviolo, A; Siracusa, P; Gennari, F. y Corso, H. (2004) sobre didáctica de las disoluciones, expresan que un aspecto de difícil interpretación, en cualquier nivel escolar, tiene que ver con las disoluciones, sobre todo en lo que se refiere a la relación entre cantidades de soluto y solvente. Presentan un modelo analógico de puntos para explicar dicha relación, similar al desarrollado por
McElroy (1996), quien diseña el modelo para mejorar la comprensión y el cálculo matemático del concepto de concentración en estudiantes de química de nivel universitario.

Este estudio se centró en identificar y construir algunas explicaciones sobre los modelos epistemológicos construidos por los profesores, en formación, a partir de las aproximaciones que hicieron (Aduríz-Bravo e Izquierdo, 2002) sobre las publicaciones histórica y epistemológicamente válidas, únicamente para las disoluciones electrolíticas en medio acuoso. Dentro del entramado conceptual de la estructuración cognoscitiva disciplinar, ha sido escasa la exploración que se ha hecho de este aspecto, respecto de la incidencia que pueda tener sobre los profesores en formación inicial en química, (Raviolo, Siracusa, Gennari y Corso, 2004). Adicionalmente, dentro de la literatura indagada, no se encontraron trabajos que estudien los aportes históricos y epistemológicos de las ciencias al desarrollo de la estructura epistemológica sobre las disoluciones en general, o de las disoluciones electrolíticas en particular.

Para Nappa, Insausti y Sigüenza, (2005) la construcción de representaciones mentales sobre las disoluciones, implica una propuesta más amplia, que abarque el cálculo de la concentración de las disoluciones y la inclusión de conceptos tales como mezclas, sistemas homogéneos y heterogéneos, sustancias, elementos, compuestos entre otros. Por otra parte, no se hallaron registros acerca del vínculo entre reconstrucciones epistemológicas e históricas sobre los modelos que se admiten para la enseñanza y aprendizaje de las disoluciones 
electrolíticas en general. Por tanto, es desde este aspecto particular que se soportó la construcción o modificación de los modelos que evidenciaron los participantes.

\section{Metodología}

En este estudio se desarrolló un revisión de referentes teórico en relación con la intervención didáctica con una metodología de entrada- salida, cuya evaluación se da en términos de trasformaciones, en un grupo de 23 profesores de química en formación inicial, de la carrera de Licenciatura en Química de la Universidad Pedagógica Nacional.

La metodología aplicada para este estudio se ajustó alrededor de tres aspectos: ¿Qué modelos han elaborado los profesores de química en formación inicial sobre disoluciones electrolíticas?, ¿una estrategia centrada en lo históricoepistemológico de modelos sobre disoluciones electrolíticas, propicia una aproximación de los profesores de química en formación inicial a lo admitido por la comunidad de químicos? ¿Son los modelos propuestos por los profesores de química, acerca de disoluciones electrolíticas, un punto de partida adecuado para la transformación en términos de una aproximación a lo aceptado por la comunidad de químicos?

De acuerdo con los propósitos de búsqueda, y la convicción de que el trabajo en el aula necesita de una visión menos tradicional, se inició la estrategia con la identificación de los modelos, sobre disoluciones electrolíticas, de sentido común que habían elaborado los profesores de química en formación inicial. Éstos se sometieron a factores de modificación, como lectura de escritos de importancia histórico-epistemológi- ca, elaboración y análisis de modelos analógicos con posterior convalidación de los mismos ante el colectivo, reproducción de experimentos históricamente determinantes como espacio para poner a prueba los modelos que hasta ese momento se hubieran construido.

El modelo metodológico propuesto (Gráfico 1), fue convalidado por especialistas en el campo de la didáctica durante el IV Congreso Iberoamericano de Educación Científica en Lima-Perú (2006).

La transformación de dichos modelos se estableció mediante el análisis del trabajo individual, o por pequeños grupos, la discusión de las aproximaciones hechas a nivel individual y colectivo, y la reelaboración de los discursos escritos. Se utilizaron dos formas para reunir la información, teniendo en cuenta que el trabajo fue desarrollado en principio de manera individual y luego se desarrolló grupalmente. Como estrategia de triangulación de los datos se tuvieron en cuenta resultados obtenidos durante las conversaciones grupales, los trabajos de interpretación individuales y grupales y las modificaciones representacionales evidenciadas como una acción que depende de lo que se piense sobre lo que se hace y lo que se dice. Los instrumentos individuales que se tuvieron en cuenta fueron: la indagación semántica, primera composición sobre disoluciones en general, primeros cuestionamientos de lápiz y papel y conversatorios. Los instrumentos colectivos que se tuvieron en cuenta fueron: segunda composición sobre disoluciones electrolíticas y disoluciones ácido-base, construcción de mapas conceptuales, cuestionamientos de lápiz y papel, ensayos sobre problemas experimentales y conversatorios. 


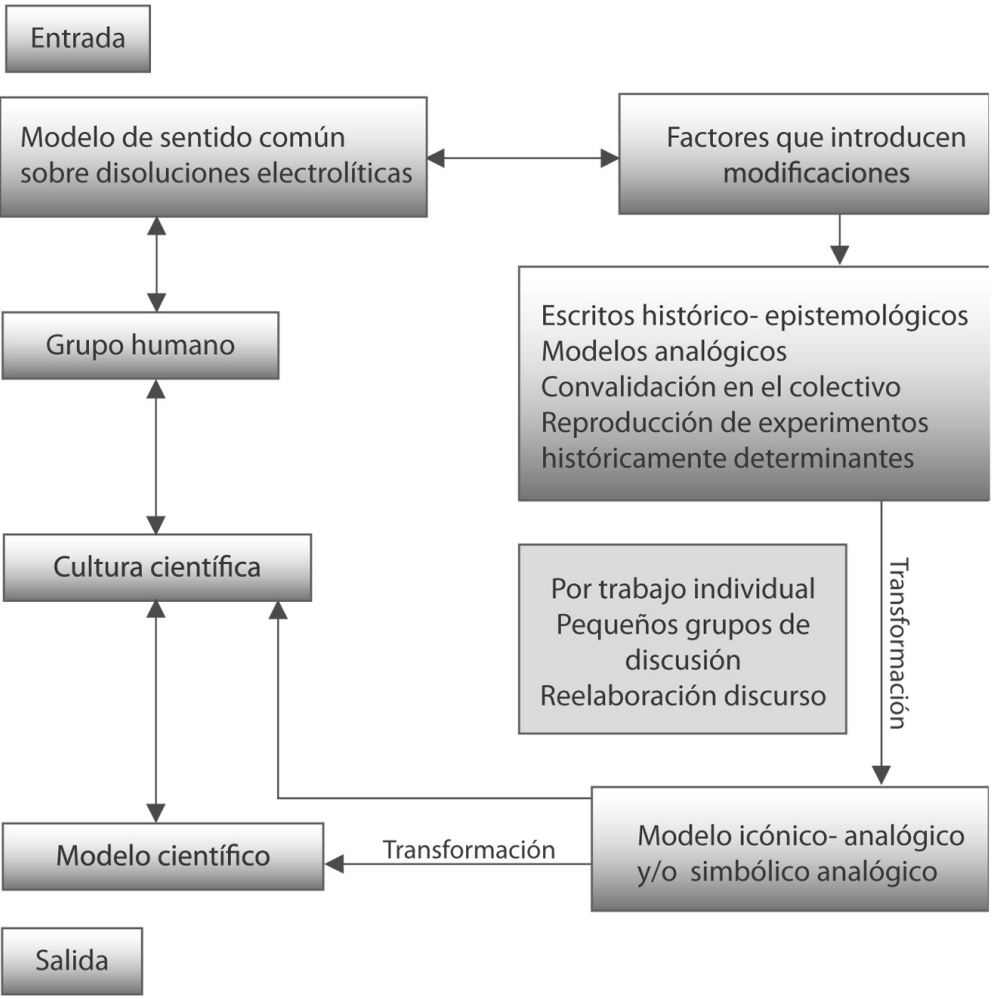

Gráfico 1. Modelo de la estrategia didáctica.

Observándose concomitantemente el cambio en la cultura científica del grupo humano participante.

Los criterios seleccionados para tratar la información colectada se centraron en los siguientes aspectos:

- Uso adecuado de la terminología sobre disoluciones electrolíticas, por dar cuenta de modelos lingüísticos significativos dentro de las relaciones de los modelos científicos.

- Tipo de relaciones evidenciadas al relacionar modelos elaborados para construir explicaciones admisibles para el tema en cuestión.
. Los distintos ambientes creados, identificando si facilitan o no una aproximación a los modelos consensuados por la comunidad de químicos sobre la propuesta histórica-epistemológica de las disoluciones electrolíticas, de acuerdo con los niveles de distinción establecidos para la categorización de los mismos.

- La categorización de los modelos se hizo de acuerdo con la siguiente clasificación construida a partir de las propuestas hechas para modelos químicos de Tomasi (1999) y Caldín (2002).

- A partir de las propuestas hechas por Tomasi (1999) y Caldín (2002) para modelos químicos, se hizo una catego- 
rización de los modelos identificados en los estudiantes, partiendo del siguiente esquema:

\section{Modelos Interpretativos * * (MI):}

Simbólico: (Sim) matemático

Material o físico (Ma): objeto de la realidad

Analógico (An): comparación

\section{Modelos Descriptivos * * (MD):}

Lingüístico (Li): término específico

Icónico (Ic): Dibujos

**Los niveles de distinción que se tuvieron en cuenta se referencian así:

S. Simplicidad: sencillo pero comple-

to, reducido.

C. Consistencia en sí mismo: permite explicar según sistema teórico admitido.
E. Estabilidad: se puede modificar sin perder estructura.

G. Generalidad: comparte propiedades con modelos no considerados inicialmente.

U. Utilidad: acorde con el sistema teórico, permite elaborar explicaciones

La anterior categorización se propuso teniendo en cuenta las características que se pueden identificar en la red de modelos que ha sido admitida por la comunidad de especialistas para construir explicaciones admisibles sobre el comportamiento de las disoluciones. A continuación se presentan las matrices diseñadas para el análisis de la in f or mación.

\begin{tabular}{|c|c|c|c|c|c|c|c|c|c|}
\hline \multirow[t]{2}{*}{ PQFI } & \multicolumn{3}{|c|}{ Modelo Descriptivo** } & \multicolumn{5}{|c|}{ Nivel de distinción *** } & \multirow[t]{2}{*}{ Observaciones } \\
\hline & LC & & LI & S & C & E & G & & \\
\hline \multicolumn{10}{|l|}{1} \\
\hline \multicolumn{10}{|l|}{2} \\
\hline \multirow[t]{2}{*}{ PQFI } & \multicolumn{3}{|c|}{ Modelo Interpretativo* } & \multicolumn{5}{|c|}{ Nivel de distinción ${ }^{* * *}$} & Observaciones \\
\hline & Sim & $\mathrm{Ma}$ & $\mathrm{An}$ & $\mathrm{S}$ & $\mathrm{C}$ & $E$ & $G$ & $\mathrm{U}$ & \\
\hline 1 & & & & & & & & & \\
\hline 2 & & & & & & & & & \\
\hline
\end{tabular}

Tabla 1. Matrices de análisis de modelos elaborados por Profesores de Química en Formación Inicial (PQFI).

\section{Resultados}

Los modelos con que ingresan los profesores de química en formación inicial respecto de las disoluciones en general y las disoluciones electrolíticas en particular, son principalmente de carácter lingüístico e icónico, si bien utilizan términos que hacen referencia a modelos científicos del campo disciplinar, en el uso se denota un sentido común.

\section{Ejemplo 1}

"Las características físicas tienen que ser observables en una mezcla o disolución para mí sería el soluto y el solvente".

Esta es una representación del uso de modelos eminentemente lingüísticos de sentido común.

El siguiente ejemplo, es una muestra de otro tipo de modelos elaborados en la misma instancia de desarrollo metodológico. 


\section{Ejemplo 2}

2. Una mezcla de naturaleza homogénea o heterogénea, donde hay un soluto y un solvente.

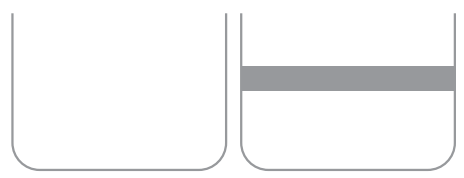

3. La concentración es una medida de la cantidad de la sustancia.

Trascripción del documento escrito por un estudiante.

Como se observa en el ejemplo 2, los modelos icónicos no se complejizan con los modelos lingüísticos, muestran confusión en los modelos lingüísticos necesarios para el tema que nos ocupa.

Luego de iniciar la aproximación histórica y epistemológica, utilizando publicaciones representativas sobre las disoluciones electrolíticas, las composiciones escritas elaboradas en forma individual muestran el uso de modelos lingüísticos relacionados con el modelo material que se está analizando. Aunque no hay utilización de modelos más elaborados y por tanto más científicos, se nota una variación sustancial con respecto a la prueba semántica inicial, se observó además, que utilizan las analogías para mejorar la explicación. En el ejemplo 3, que se muestra a continuación, hay un fragmento escrito que muestra cómo se van relacionando de manera más compleja y menos confusa los modelos lingüísticos seleccionados.

Estos escritos fueron elaborados de manera individual antes de una sesión de socialización sobre la temática propuesta.

\section{Ejemplo 3}

Soluciones y sus propiedades

Las soluciones son muy importantes para todos los procesos vitrales, por ejemplo la orina en los animales, la savia en las plantas, y sobre todo la gran mayoría de las sustancias que existen en la tierra, se encuentran en solución. Se denomina soluto a la sustancia disuelta y solvente al medio en que se disuelve. Un tipo importante de soluto es el electrolito que se disocia par dar iones a la solución. Estos se pueden clasificar en fuertes y débiles, según el grado de disolución. Las soluciones pueden conducir la electricidad o no dependiendo de las sustancias con que se encuentran mezcladas.

Cuando una solución, es acuosa, o sea que se encuentra disuelta con el agua, se puede disociar electrolíticamente.

Según Svante Archenius, todos los ácidos, tienen hidrógeno y las propiedades cíclicas, están sujetas a la presencia de átomos de hidrógeno ionizable. En la misma forma, todas las bases, tienen hidróxidos y las propiedades básicas se atribuyen y que al cambiarse entre si, producen una neutralización para formar una molécula de agua.

¿Pero esta teoría daría una verdadera definición tanto en el concepto como en sus propiedades a los ácidos y a las bases?

Pues no, esta teoría hecha por Archenius fue útil aunque presentaba ciertas desventajas, la primera, soluciones acuosas, la segunda ignoraba sustancias que se disuelven en iones diferentes $\mathrm{OH}$ - que se combinan con $\mathrm{H}+\mathrm{y}$ viceversa" (Trascripción de una de las respuestas presentadas por un grupo de estudiantes). 
En una segunda instancia, las composiciones analizadas corresponden a resultados del trabajo colectivo, luego de una socialización al interior del grupo. Los modelos identificados en los documentos producidos en ese momento se clasifican como del tipo simbólicoanalógico, si bien se evidencia el uso de modelos lingüísticos e icónicos, estos son más consistentes por las relaciones entre unos y otros.

\section{Ejemplo 4}

Claro que la propuesta acido-básica mas completa fue presentada en 1923 por el profesor G. N. Lewis.

Según él, un ácido, es una especie que puede aceptar y compartir un par de electrones y una base, es cualquier especie que puede o es capaz de ceder y compartir un par de electrones esta teoría en general abarcó todas las relaciones acido-básicas de otras teorías y otras reacciones adicionales.

Teoría Wransted

$\underset{\text { acido }_{1}}{\mathrm{H}_{2} \mathrm{O}}+\underset{\text { base }_{1}}{\mathrm{H}_{2} \mathrm{O}} \Longleftarrow \underset{\text { acido }_{2}}{\mathrm{H}_{3} \mathrm{O}^{+}}+\underset{\text { base }_{2}}{\mathrm{OH}^{\prime-}}$

Teoría Lewis

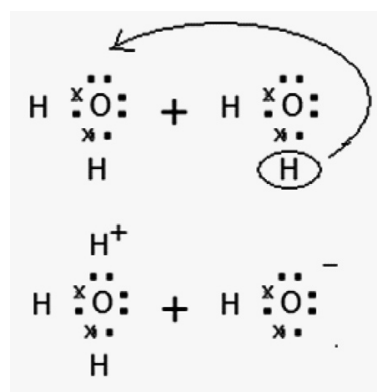

Trascripción del documento escrito por un grupo de estudiantes.
Fragmento de una composición sobre la evolución histórica epistemológica de ácidos y bases construida en colectivo.

Se aclara que el discurso oral, también fue objeto de análisis por mostrar hasta qué punto, los modelos lingüísticos evidenciados en otras instancias hacían parte real de las estructuras epistemológicas en construcción.

Las siguientes graficas, muestran las comparaciones numéricas resultantes de la caracterización de las particularidades de los documentos, construidos a lo largo del tiempo en que se implementó la estrategia diseñada para este estudio, determinados por los diferentes niveles de distinción en las pruebas más representativas.

Se enfatiza que los análisis de los modelos y su consecuente categorización se hizo usando las matrices explicadas anteriormente, donde se hizo un seguimiento a cada participante de los modelos reelaborados en las diferentes instancias de intervención.

En las composiciones escritas, los niveles de distinción identificados en los modelos que se pudieron establecer con esta información, muestran que los modelos interpretativos, por ser más sencillos, les fueron más útiles y que la consistencia, generalidad y estabilidad se centró en los modelos descriptivos, lo que permite pensar que la importancia de las modificaciones establecidas en esta parte del proceso está relacionada con modelos descriptivos del fenómeno, sin embargo, al separar los modelos por categorías, no dicen gran cosa del proceso. 


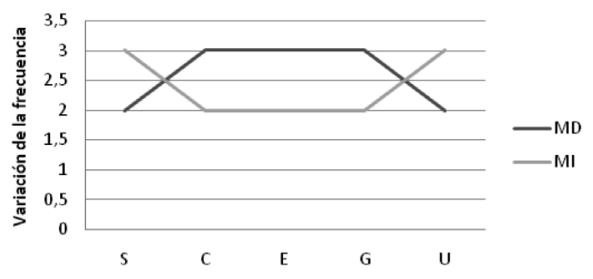

\begin{tabular}{cccccc}
\hline & \multicolumn{3}{c}{ Nivel de Distinción } & & Modelo \\
\hline S & C & E & G & U & \\
\hline 2 & 3 & 3 & 3 & 2 & MD \\
\hline 3 & 2 & 2 & 2 & 3 & MI \\
\hline
\end{tabular}

Grafico 3. Comparación de los niveles de distinción para los modelos identificados en las composiciones sobre ácido-base.

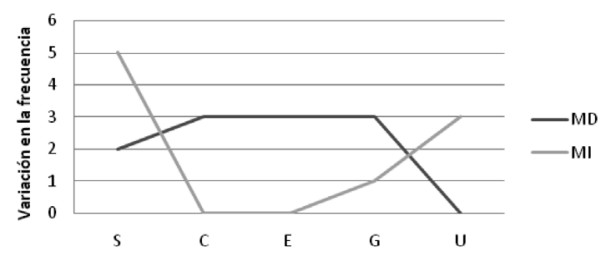

\begin{tabular}{cccccc}
\hline & \multicolumn{3}{c}{ Nivel de Distinción } & & Modelo \\
\hline S & C & E & G & U & \\
\hline 2 & 3 & 3 & 3 & 0 & MD \\
\hline 5 & 0 & 0 & 1 & 3 & Ml \\
\hline
\end{tabular}

Grafico 4. Comparación de los niveles de distinción para los modelos identificados en los mapas conceptuales elaborados de forma grupal.

$\mathrm{Al}$ analizar los mapas conceptuales, con respecto a los modelos que sobre disoluciones electrolíticas construyeron de manera individual, se observó un amplio nivel de simplicidad en las estructuras, hecho que cambió sustancialmente al trabajar en el colectivo. Se puede afirmar que la discusión entre pares mejoraba significativamente los resultados, mostrando al final modelos con fuertes relaciones entre sí que se evidenciaron en la jerarquización de los términos que referenciaron los diferentes modelos científicos implicados en la explicación del comportamiento de disoluciones electrolíticas en medio acuoso.

La grafica comparativa corrobora lo dicho; los modelos lingüísticos son el soporte del trabajo y por lo tanto, los modelos interpretativos, ofrecen, desde su simplicidad, provecho, aunque no soporten modificaciones debido a que son poco estables.

En lo concerniente a cuestionamientos de lápiz y papel, se notó al comienzo, una tendencia a minimizar las explicaciones a lo estrictamente simbólico, esto conduce a pensar que dentro de la experiencia educativa anterior, este aspecto fue ampliamente reforzado como el más importante, por lo tanto, los modelos con los que entran al proceso de formación docente, son de carácter descriptivo (icónico- analógico). Dichos modelos no son de gran utilidad a los profesores en formación cuando buscan explicaciones del comportamiento de las disoluciones electrolíticas en diferentes condiciones.

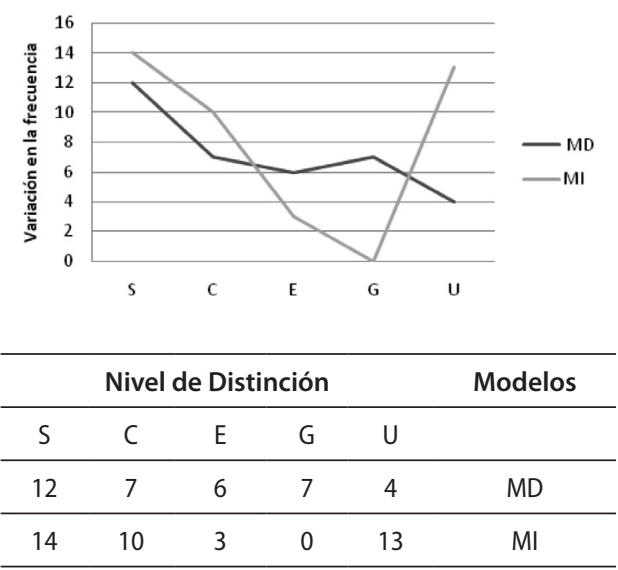

Grafico 5. Comparación de los niveles de distinción para los modelos identificados en los cuestionamientos de lápiz y papel (trabajo individual). 
Luego de una aproximación históricoepistemológica y de un trabajo en colectivo, en una segunda oportunidad de trabajo con cuestionamientos de lápiz y papel, el cambio observado es sustancial, puesto que la explicación se hace compleja en el ejercicio de mostrar las relaciones entre los modelos simbólicos y los modelos materiales (analógico-simbólico), la interpretación de los modelos simbólicos utilizados se muestra en los modelos lingüísticos desarrollados.

\section{Ejemplo 5}

$$
\begin{aligned}
& 2 \overline{68} \mathrm{~g} \quad 5464,8 \mathrm{~g} \quad 686 \mathrm{~g} \\
& \mathrm{Na}_{2}^{11} \mathrm{Cr}_{2}^{16} \mathrm{O}_{7}^{-2}+6 \mathrm{Fe}^{12} \mathrm{~S}^{+6} \mathrm{O}_{4}^{-2}+7 \mathrm{H}_{2}^{11} \mathrm{~S}^{16} \mathrm{O}_{4}^{-2} \rightarrow \\
& 0,2 \mathrm{~N} \quad 0,76 \mathrm{gr} \quad 0,1 \mathrm{M} \\
& \begin{array}{l}
1198,8 g \\
3 \mathrm{Fe}_{2}^{13}\left(\mathrm{~S}^{16} \mathrm{O}_{4}^{-2}\right)_{3}{ }^{+}{ }^{+} \mathrm{Na}_{2}^{11} \mathrm{~S}^{+6} \mathrm{O}_{4}^{-2}{ }^{+} \mathrm{Cr}_{2}^{13}\left(\mathrm{~S}^{11} \mathrm{O}_{4}^{-2}\right)_{3}{ }^{+} \ldots
\end{array} \\
& \frac{e q-g}{L s l N}=N \\
& 262 \mathrm{gNa}_{2} \mathrm{Cr}_{2} \mathrm{O}_{7} \rightarrow 5464,8 \mathrm{~g} \cdot \mathrm{FeSO}_{4} \\
& x \rightarrow 0,76 \mathrm{~g} \cdot \mathrm{FeSO}_{4} \quad x=0,36 g \cdot \mathrm{Na}_{2} \mathrm{Cr}_{2} \mathrm{O}_{7} \\
& \frac{0,36 g \cdot \mathrm{Na}_{2} \mathrm{Cr}_{2} \mathrm{O}_{7}}{14^{(1)(-1)}}=e q-g \\
& e q-g=0,00257 \\
& \frac{e q-g}{L s l N}=N \quad \frac{0,00257 e q-g}{0,2 N}=L s l N \\
& L=0,0128 \text { de } \mathrm{Na}_{2} \mathrm{Cr}_{2} \mathrm{O}_{7}
\end{aligned}
$$

Desarrollo de un modelo simbólico sobre reacciones en medio acuoso.

Con respecto a los problemas experimentales planteados a los estudiantes desde el contexto histórico-epistemológico de las disoluciones,en la intervención en el aula, se contrastan los distintos modelos elaborados por los profesores en formación sobre disoluciones electrolíticas y se establecen relaciones entre aquellos que son de tipo lingüístico con otros que son analógicos, simbólicos y materiales. En este tipo de intervención educativa se nota el inicio de la transición entre los modelos descriptivos que poseen los estudiantes, hacia modelos interpretativos mas complejos, como los analógicos-simbólicos.

En la gráfica se puede identificar que hay diferencias sustanciales entre un tipo de modelo y otro; respecto de su distinción, se confirma que, por separado, las explicaciones tienden a carecer de sentido y que son complementarios entre sí.

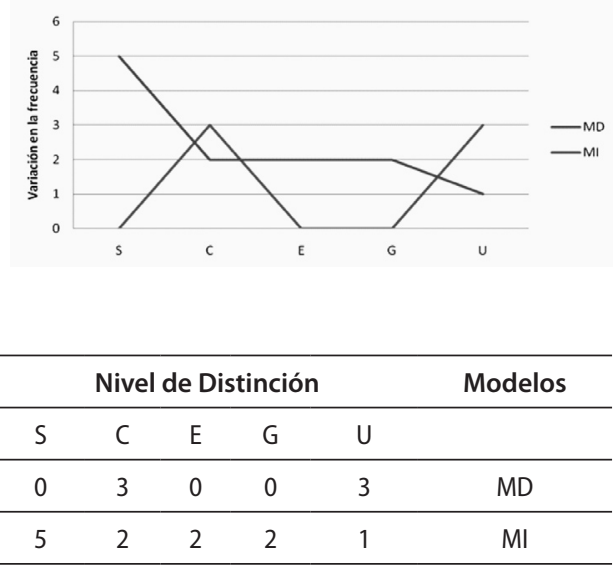

Grafico 6. Comparación de los niveles de distinción para los modelos identificados en los cuestionamientos de lápiz y papel (trabajo colectivo). 
En los conversatorios, se indagó fundamentalmente, acerca del uso de los modelos lingüísticos y de las relaciones establecidas con los modelos simbólicos, analógicos e icónicos. Durante las sustentaciones frente al grupo, se pudo notar que aunque se presentaban diferencias que servían para identificar un avance en la transformación de modelos de sentido común hacia modelos científicos, dichas modificaciones no son homogéneas, se particularizan por individuos o en pequeños grupos de trabajo.

Para finalizar, las siguientes dos gráficas ( 7 y 8 ) se elaboraron con el propósito de indicar la variación en el uso de uno u otro tipo de modelo a lo largo de la experiencia y resaltar que se presentaron cambios sustanciales, en el trabajo colectivo e individual, respecto de la construcción y modificación de modelos sobre disoluciones y disoluciones electrolíticas en particular, por parte de los participantes en este estudio.

Si bien se notaron modificaciones en la presencia de modelos icónicos a lo largo de las pruebas, se mantuvo en cada una de ellas una gran diferencia entre éstos y el uso de modelos lingüísticos, con lo que no es posible establecer el aumento o descenso progresivo de su uso a lo largo de las diferentes actividades, por considerarse que eso dependía del contexto desde el cual se indagara.

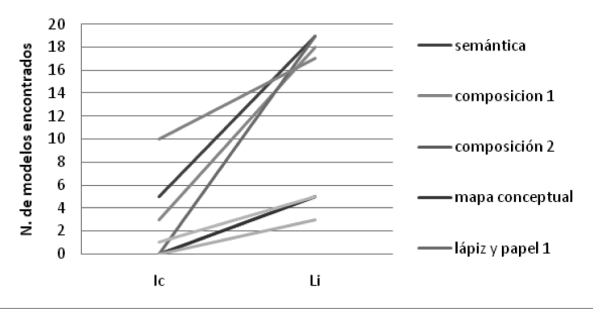

\begin{tabular}{lll}
\hline Prueba & Ic & Li \\
\hline Semántica & 5 & 19 \\
\hline Composición 1 & 3 & 18 \\
\hline Composición 2 & 0 & 5 \\
\hline Mapa conceptual & 0 & 5 \\
\hline Lápiz y papel 1 & 0 & 19 \\
\hline Lápiz y papel 2 & 0 & 3 \\
\hline $\begin{array}{l}\text { Ensayo problema } \\
\text { experimental 1 }\end{array}$ & 10 & 17 \\
\hline $\begin{array}{l}\text { Ensayo problema } \\
\text { experimental 2 }\end{array}$ & 1 & 5
\end{tabular}

Grafico 7. Tendencia del modelo descriptivo en cada una de las pruebas.

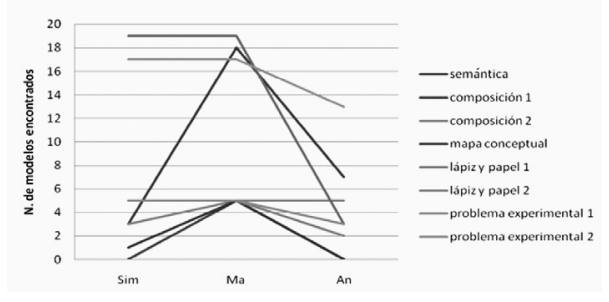

\begin{tabular}{llll}
\hline Prueba & Sim & Ma & An \\
\hline Semántica & 0 & 5 & 0 \\
\hline Composición 1 & 3 & 18 & 7 \\
\hline Composición 2 & 3 & 5 & 5 \\
\hline Mapa conceptual & 1 & 5 & 0 \\
\hline Lápiz y papel 1 & 19 & 19 & 3 \\
\hline Lápiz y papel 2 & 5 & 5 & 2 \\
\hline $\begin{array}{l}\text { Ensayo problema } \\
\text { experimental 1 }\end{array}$ & 17 & 17 & 13 \\
\hline $\begin{array}{l}\text { Ensayo problema } \\
\text { experimental 2 }\end{array}$ & 5 & 5 & 3
\end{tabular}

Grafico 8. Tendencia del modelo interpretativo en cada una de las pruebas.

Como en el caso anterior, para los modelos interpretativos, se puede decir que se observó la mayor tendencia para el modelo material. Lo que indica que el 
grupo en general puso en claro el objeto de estudio con respecto a las disoluciones electrolíticas, los modelos eran más complejos a partir del trabajo colectivo, llegando a instancias en las que se logró el manejo de modelos simbólicoanalógicos, que son en últimas, una ruta adecuada hacia los modelos científicos sobre disoluciones electrolíticas.

\section{Conclusiones}

Se puede afirmar que el proceso de modificación, evidenciado en los modelos que sobre disoluciones electrolíticas, y en la información suministrada por los profesores en formación inicial de química de la Universidad Pedagógica Nacional, que participaron en este estudio, se caracterizó por comenzar en su mayoría, con modelos de sentido común que se expresan en formas icónicoanalógicas y en la mayoría de casos con modelos lingüísticos. Se pudo establecer además, que los participantes tomaron diferentes direcciones, evidentes con el hacer complejos los modelos elaborados, lo que indica una ruta particular en el proceso de construcción o reconstrucción epistemológica para las disoluciones electrolíticas; por este motivo no es fácil establecer una caracterización única para el grupo de modelos que se pueden identificar, aunque incluyan con mayor frecuencia modelos analógicos, que pueden o no estructurarse hacia modelos simbólico- analógicos, más próximos a los modelos característicos del conocimiento científico.

Las estrategias metodológicas diseñadas, que facilitaron procesos de concertación y discusión para elaborar explicaciones admisibles a las condiciones propuestas por el investigador, muestran que no solo es importante dar relevancia a los modelos simbólicos, sino que éstos son más representativos cuando se logran establecer relaciones estrechas de significado entre diferentes clases de modelos. Por lo tanto, los distintos espacios de discusión y construcción no son determinantes por separado.

Los modelos que llegan al proceso didáctico con los profesores en formación inicial de química son indispensables, en la medida en que su caracterización facilita la elaboración de estrategias didácticas para desarrollar de manera consciente, y además permiten el proceso de transición de modelos comunes o poco elaborados hacia modelos didactizados admitidos por la comunidad de químicos, facilitando el fortalecimiento de la estructura cognitiva disciplinar a lo largo de su formación profesional.

Sobre la idea de producción social del saber científico, se puede afirmar que, a pesar de no ser el objeto de estudio de este trabajo, los participantes mostraron un cambio en sus concepciones, reconociendo la importancia de acceder a documentos de validez histórica y epistemológica, y mostrando con ello el cambio en la cultura científica del grupo humano participante.

Sobre las modificaciones de los sistemas de modelos elaborados por los participantes, se resalta el cambio de modelos cotidianos, fundamentalmente icónicos, hacia modelos icónicoanalógicos e icónico-simbólicos, que son el origen de los modelos científicos por excelencia.

Los autores agradecen especialmente a los profesores en formación que participaron desinteresadamente en este pro- 
ceso y a los profesores de la Universidad Pedagógica Nacional que aportaron en el diseño metodológico y facilitaron los espacios para su ejecución.

\section{Recomendaciones y sugerencias}

De acuerdo con lo indagado, en los primeros semestres del programa de formación inicial de profesores de Química, de conformidad con los respectivos proyectos curriculares, se ha de propiciar un ambiente didáctico que haga

\section{Referencias bibliográfícas}

Aduríz-Bravo, A.; Izquierdo, M. y Estany, A. (2002). Una propuesta para estructurar la enseñanza de la filosofía de la ciencia para el profesorado de ciencias. En Formación. Enseñanza de las Ciencias. 20 (3), 465-476.

Aduríz-Bravo, A. e Izquierdo, M. (2002). Acerca de la Didáctica de las Ciencias como disciplina autónoma. Revista Electrónica de Enseñanza de las Ciencias. 1 (3), 130-140.

Aduríz-Bravo, A.; Garófalo, J., Greco, M. y Galagovsky, L. (2005). Modelo Didáctico Analógico. Marco teórico y ejemplos. Enseñanza de las Ciencias. Número extra VII Congreso. pp.1-6.

Caldin, E. (2002). The structure of chemistry in relation to the philosophy of science. Hyle International Journal for Philosophy of Chemistry. 8 (2), 103121, http://www.hyle.org/journal/ issues/8-2/caldin.html.

D’Amoré, B. (2007). El papel de la epistemología en la formación de profesores de matemáticas de la escuela secundaria. Colección Cuadernos del Seminario en Educación. No. 8. Bogotá: Instituto de Investigación en Educación Facultad de Ciencias Humanas. Universidad Nacional de Colombia. posible la identificación, caracterización y discusión de los modelos elaborados sobre sistemas teóricos estructurantes en Química, con los cuales ingresan al proceso los profesores en formación, todo, para que haya la posibilidad de aproximar de manera admisible durante toda la carrera, los modelos construidos en otras instancias escolares distintos de los formulados y aceptados por la comunidad de especialistas, de manera que las redes de modelos se fortalezcan entre sí.

Galagovsky L.; Di Giacomo M. y Castelo V. (2009). Modelos vs. dibujos: el caso de la enseñanza de las fuerzas intermoleculares, Revista Electrónica de Enseñanza de las Ciencias, 8(1), obtenido el día 28 de febrero de 2009 desde http://www.saum.uvigo.es/reec/

Gallego Badillo, R. y Pérez Miranda, R. (2003). El problema del cambio en las concepciones epistemológicas, pedagógicas y didácticas. Bogotá: Universidad Pedagógica Nacional. Facultad de Ciencia y Tecnología. Departamento de Química.

Gallego Badillo, R. (2004). Un concepto epistemológico de modelo para la didáctica de las Ciencias Experimentales. Revista Electrónica de Enseñanza de las Ciencias. 3(3).76-95

Gallego Badillo, R.; Pérez Miranda, R., Gallego Torres, A. P. y Torres de Gallego L. (2007). El objeto de saber de los químicos. Formulación, modificación y abandono del modelo icónico inicial. En Investigações em Ensino de Ciências, 11(3). Porto Alegre, Brasil: Instituto de Física, Universidade Federal do Rio Grande do Sul. Extraído de: http://www.if.ufrgs.br/public/ ensino/vol11/n3/v11_n3_a5.htm 
Giere, R. N. (1992). La explicación de la ciencia. Un acercamiento cognoscitivo. Colección Ciencia Básica. México: Consejo Nacional de Ciencia y Tecnología.

Gil Pérez, D.; Carrascosa Alís, J. y Martínez Terrades, F. (1999). El surgimiento de la didáctica de las ciencias como campo específico de conocimientos. Educación y Pedagogía. 11(25). 15-47.

Greca, I. M. y Dos Santos, F. M. T. (2005). Dificuldades da generalização das estratégias de modelaçãoemciências: O caso da física e da química. Investigações em Emsino de Ciências. Extraído de http://www.if.ufrgs.br/public/ ensino/vol10/n1/v10_n1_a2.htm

Kuhn T. S. (2000). La estructura de las Revoluciones Científicas. Bogotá: Fondo de Cultura Económica.

Lombardi, O. (1998). La noción de modelo en ciencias. Educación en Ciencias, Vol. II, No. 4, 5-13.

McElroy, L. (1996). Teaching Dilutions. Journal of Chemical Education. 73(8), 765-766.

Nappa, N.; Insausti, M. y Sigüenza, A. (2005). Obstáculos para generar representaciones mentales adecuadas sobre la disolución. Revista Eureka sobre la enseñanza y divulgación de las ciencias. 2(3), 344-363.

Niaz, M. (2009). Progressive transitions in chemistry teachers' understanding of nature of science based in historical controversias. Science \& Education, obtenido el día 30 de Julio desde http://www.springerlink.com/ content/963w615367027262/

Pessoa De Carvalho, A. (2004). Formación de profesores: es necesario que la didáctica de las ciencias incluya la práctica de la enseñanza. Educación Química. 15(1), 15-22.

Raviolo, A.; Siracusa, P.; Gennari, F. y Corso, H. (2004). Utilización de un modelo analógico para facilitar la comprensión del proceso de preparación de disoluciones. Primeros resultados. Enseñanza de las Ciencias. 22(3), 379-388.

Tomasi, J. (1999). Towards' chemical congruence' of the models in theoretical chemistry. En HYLE International Journal for the Philosophy of Chemistry. 5(2),79-115, Extraído de http://www. hyle.org/journal/issues/5/tomasi. htm 